Stability Analysis of Finite-difference Schemes for the Viscoelastic Wave Equation

Joakim O. Blanch

William W. Symes

October, 1994

TR94-35 



\title{
Stability Analysis of Finite-difference Schemes for the Viscoelastic Wave Equation
}

\author{
Joakim O. Blanch* William W. Symes ${ }^{\dagger}$
}

October 5, 1994

\begin{abstract}
It is difficult to predict stability properties of a finite difference scheme. It has to be investigated through the roots of the $Z$-transformed and Fourier transformed difference scheme (modal equation). To simultaneously investigate several schemes for the viscoelastic wave equation, it is possible to derive the modal equation with parameterized coefficients. Several conditionally stable schemes were found, where the most efficient is a staggered scheme with a stability condition closely resembling that of an elastic scheme.
\end{abstract}

\section{Introduction}

Finite difference schemes are often used to numerically solve ordinary and partial differential equations, [7]. It is easy to create such a scheme, since derivatives are simply exchanged for difference ratios, which approximate the derivatives to some accuracy. The accuracy is found through Taylor expansion. There is no standard rule for how these schemes are created and thus

*The Rice Inversion Project, Department of Geology and Geophysics, Rice University, Houston TX 77251-1892

${ }^{\dagger}$ The Rice Inversion Project, Department of Computational and Applied Mathematics, Rice University, Houston TX 77251-1892 
leaves quite some room for experimentation. The accuracy of the finite difference scheme is easy to find and it is commonly a good measure for how well the scheme will solve the differential equation, i.e., the higher the accuracy, the closer the numerical solution will be to the true solution. For time dependent problems the solution at earlier time is used to update the solution to the next time level. There are two distinctive techniques to update the solution in time, explicit or implicit updating (time stepping). For implicit time stepping it is necessary to solve an equation system to update the solution in time. Explicit time stepping is thus naturally faster. Implicit time stepping tends to attenuate higher frequency modes in the solution as well. Explicit time stepping is always unstable (solution grows uncontrollably) for at least some choices of parameters, e.g., time step, whereas implicit time stepping is stable (for well-posed problems). Explicit time stepping is preferred for hyperbolic problems, since it does not necessarily attenuate the solution (hyperbolic systems are energy preserving) and is fast. Hence, it is necessary to find the parameter choices for which the explicit scheme is not unstable, i.e. the stability condition. This can prove difficult since some schemes are unconditionally unstable (no such choices exist) and only some conditionally stable. The problem is thus to find a conditionally stable scheme and the stability condition.

It is possible to investigate the stability of a particular finite difference scheme by Fourier transforming the scheme in space and $Z$-transforming the scheme in time. The result is an equation which depends on wavenumber. The scheme is stable if the absolute value of the roots (poles of the scheme) of the equation are all equal to or less than one for all possible wavenumbers [7]. The more strict formulation states: It is an necessary condition for stability or It is a sufficient condition for instability IF the absolute value of one or more of the poles is greater than one. The method implicitly implies that parameters for the differential equation are independent of space (constants).

Some initial work to solve the viscoelastic wave equation with an explicit finite difference scheme encountered stability problems, [1], [2]. To find a reliable scheme it was clearly necessary to thoroughly investigate the stability properties of several different realizations. All the realizations are first or second order accurate in time and fourth order accurate in space and based on a stress-velocity formulation with one memory variable, [1], [9].

The differential equation and the several different finite-difference scheme realizations will be covered in the first section as well as the investigation 
method. The following section presents the investigation and stability results for the schemes. Finally, the most efficient conditionally stable scheme will be thoroughly analyzed for its stability condition.

\section{Viscoelastic Wave Propagation, Discrete Schemes and Method for Investigation}

The 1-D viscoelastic wave equation for a constitutive relation corresponding to a standard linear solid is,

$$
\left\{\begin{array}{l}
p_{, t}=-K(1+\tau) v_{, x}-r \\
r_{, t}=-\frac{1}{\tau_{\sigma}}\left(r+K \tau v_{, x}\right) \\
v_{, t}=-\frac{1}{\rho} p_{, x}
\end{array},\right.
$$

[5]. It is an initial value problem, i.e., $p=r=v=0, t \ll 0$. $K$ is the bulk modulus, $\rho$ is the density, and $\tau$ is roughly proportional to the reciprocal of $\mathbf{Q}$ and a measure of attenuation, [5]. $\tau_{\sigma}$ is a relaxation time and determines the frequency range where there is most attenuation. $p$ is the pressure (stress) and $v$ is the particle velocity. $r$ is the, so called, memory variable, which reflects the 'memory' of the viscoelastic medium.

Several different types of schemes and grids can be used to discretize the continuous system, Equation (1). The general scheme created here can be first or second order accurate in time and fourth order accurate in space. It is possible to fit the scheme for some instances of parameter values on a staggered grid. Acoustic/elastic problems have generally been solved with leap-frog schemes for updating in time [8]. Hence, leap-frog stencils will be used for to update $p$ and $v$. The equation for the memory variable $r$ might for small values of $\tau_{\sigma}$ be stiff and it is natural to solve the equation with an Euler-Backward or Crank-Nicolson scheme, cf., [6]. With these specification a fairly general finite difference scheme for Equation (1) can be constructed. 


$$
\left\{\begin{aligned}
p_{m}^{n+1}= & p_{m}^{n-1}-\frac{\Delta t}{\Delta x} K(1+\tau) D_{0, x} v_{m}^{n}- \\
& 2 \Delta t\left(c_{1} c_{2} r_{m}^{n+1}+\left(1-c_{1}\right) r_{m}^{n}+c_{1}\left(1-c_{2}\right) r_{m}^{n-1}\right) \\
r_{m}^{n+1}= & r_{m}^{n-\beta}-(1+\beta) \frac{\Delta t}{\tau_{\sigma}}\left(\left(a r_{m}^{n+1}+(1-a) r_{m}^{n-\beta}\right)+\right. \\
& \left.\frac{K \tau}{2 \Delta x} D_{0, x}\left(b_{1} b_{2} v_{m}^{n+1}\left(1-b_{1}\right) v_{m}^{n} b_{1}\left(1-b_{2}\right) v_{m}^{n-1}\right)\right) \\
v_{m}^{n+1}= & v_{m}^{n-1}-\frac{\Delta t}{\Delta x \rho} D_{0, x} p_{m}^{n}
\end{aligned}\right.
$$

where $n$ corresponds to time level $(t=n \Delta t)$ and $m$ to space $(x=m \Delta x)$. $D_{0, x}$ is a discrete centered spatial differentiation operator. The parameters $\beta, a, b_{1}, b_{2}, c_{1}, c_{2}$ are used to change the scheme, $\beta \in\{-1,0\}, a, b_{1}, b_{2}, c_{1}, c_{2} \in$ $[0,1]$. The parameters do not affect the purely acoustic part of the scheme, but only the part connected to the memory variable. An abundance of realizations are clearly possible even though the possibilities are restricted through the small parameter space, $\beta, a$ etc.. To investigate the stability of the different realizations, the scheme [Equation (2)] is Fourier transformed in space and $Z$-transformed in time, creating a fifth or sixth order equation.

$$
\begin{aligned}
& z^{5+\beta}+z^{4+\beta} q_{r} \psi^{2} \tau b_{1} b_{2} c_{1} c_{2}-z^{4} q+ \\
& z^{3+\beta}\left[-2-\psi^{2}\left(1+\tau\left(1+q_{r}\left(b_{1} b_{2}\left(1-c_{1}\right)+c_{1} c_{2}\left(1-b_{1}\right)\right)\right)\right)\right]+ \\
& z^{2+\beta}\left[\psi^{2} \tau q_{r}\left(\left(1-b_{1}\right)\left(1-c_{1}\right)+b_{1} b_{2}\left(1-c_{2}\right)+c_{1} c_{2}\left(1-b_{2}\right)\right)\right]+ \\
& z^{2}\left[2 q-\psi^{2}(1+\tau) q\right]+z^{1+\beta}\left[1-\psi^{2} \tau q_{r}\left(\left(1-b_{1}\right) c_{1}\left(1-c_{2}\right)+\left(1-c_{1}\right) b_{1}\left(1-b_{2}\right)\right)\right]+ \\
& z^{\beta}\left[-\psi^{2} \tau q_{r} b_{1}\left(1-b_{2}\right) c_{1}\left(1-c_{2}\right)\right]-q \\
& \quad=0
\end{aligned}
$$

where $\psi^{2}=4 \lambda^{2}\left(\frac{\sin (k \Delta x)(4-\cos (k \Delta x))}{3}\right)^{2}, \quad q=\frac{1-a \eta}{1+a \eta}, q_{r}=\frac{\eta}{1+a \eta}, \quad \eta=(1+$ $\beta) \frac{\Delta t}{\tau_{\sigma}}, \lambda=c_{0} \frac{\Delta t}{\Delta x} . \quad c_{0}=\sqrt{\frac{K}{\rho}}$ is the zero frequency velocity and $k$ is the wavenumber. $\psi$ depends on the spatial accuracy of the scheme and the particular stencil. If the absolute value of the solutions to Equation (3) is less than one, then the corresponding scheme is stable. 


\section{Stability Investigation}

We will investigate three suites of different schemes. The three suites are distinguished by the values of $\beta$ and $a$. These parameters changes the stencil for updating the memory variable in time. The three different combinations are: $(\beta=0, a=1)$ (Euler backward), $(\beta=0, a=0.5)$ (Crank-Nicolson), and $(\beta=1, a=0.5)$ (Crank-Nicolson).

The parameters $b_{i}, c_{j} ; i, j \in\{1,2\}$, determines the "mix" in time of the velocity into the memory variable update equation and of the memory variable into the pressure update equation.

The original scheme by Blanch et al., [1], [2], was equivalent to $\beta=0$, $a=0.5, b_{1}=0.5, b_{2}=1$, and $c_{1}=0$. The scheme is unconditionally unstable. Figure 1 shows the position of the poles for the scheme as well as other related schemes with $\beta=0$, which results in a fifth order equation and five poles. A first attempt to adjust the scheme might be to change the source term for the memory variable equation. Changing $b_{1}$ between 0 and 1 and tracking the maximum absolute value of all the poles yields the graph in Figure 2. Changing $a$ to $a=1$ to create an even more stable scheme (Euler backward) for updating the memory variable in time has no effect on the stability for the whole scheme, Figure 3 . Changing $c_{1}$ with $a=0.5, b_{1}=0.5$, $b_{2}=1$, and $c_{2}=0.5$ produces the curve in Figure 4 . Figure 5 shows the position of the pole which causes the instability of the scheme for small values of $c_{1}$. It is clearly possible to create a conditionally stable scheme for the configuration $\beta=0$ and $a=0.5$. There is not even an $a d$ hoc explanation for how and/or why the different schemes are stable/unstable, though. Both $b_{1}$ and $c_{1}$ can be varied to create a 2 -variable plot to yield more insight into the phenomenom, Figure 6 . Stable schemes occur for large values of $b_{1}$ and $c_{1}$, suggesting that the most recent information should be used. The stable schemes are unfortunately only first order accurate, though. The only second order accurate scheme occurs for $b_{1}=c_{1}=0.5$, which is unstable.

The last suite of schemes $(\beta=1, a=0.5)$ are for some instances possible to implement as staggered schemes. Fixing $b_{2}=c_{2}=0.5$ will ensure that the schemes all are second order accurate in time for all values of $b_{1}, c_{1}$. The positions of the poles for this suite of schemes are plotted in Figure 7. The absolute value for the maximum pole as a function of $b_{1}$ and $c_{1}$ is plotted in Figure 8 and 9 . Figure 10 shows a slice from Figure 8 for $c_{1}=1$. Clearly there are several schemes which are conditionally stable. 


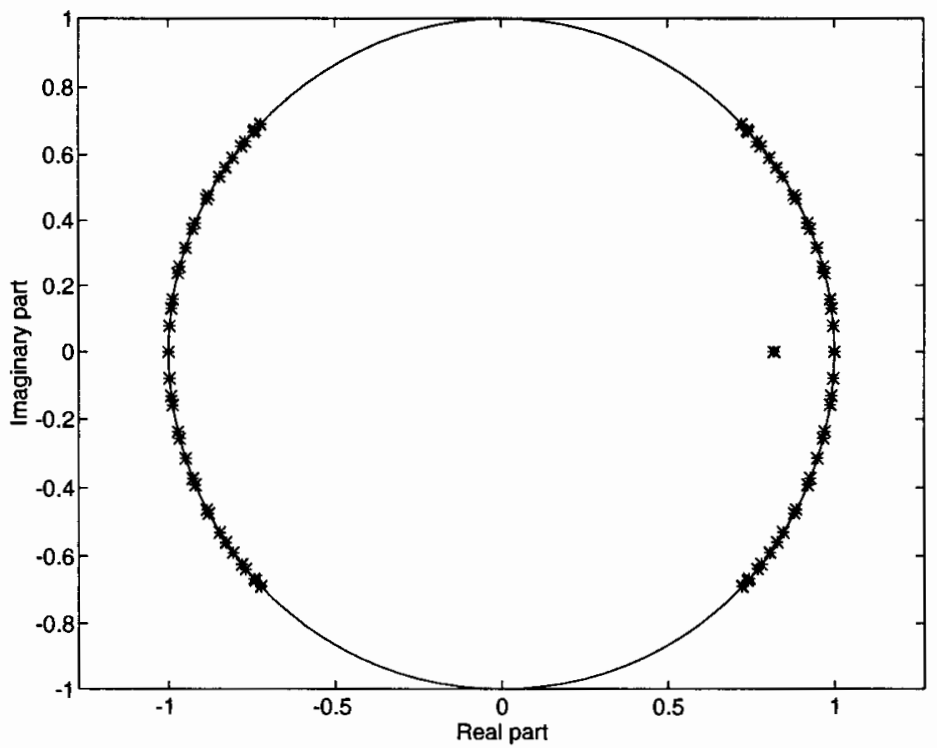

Figure 1: Position of poles for $\beta=0$ schemes. Unstable, though not obvious. It is always the poles close to -1 , which are outside the unit circle for the unstable schemes. 


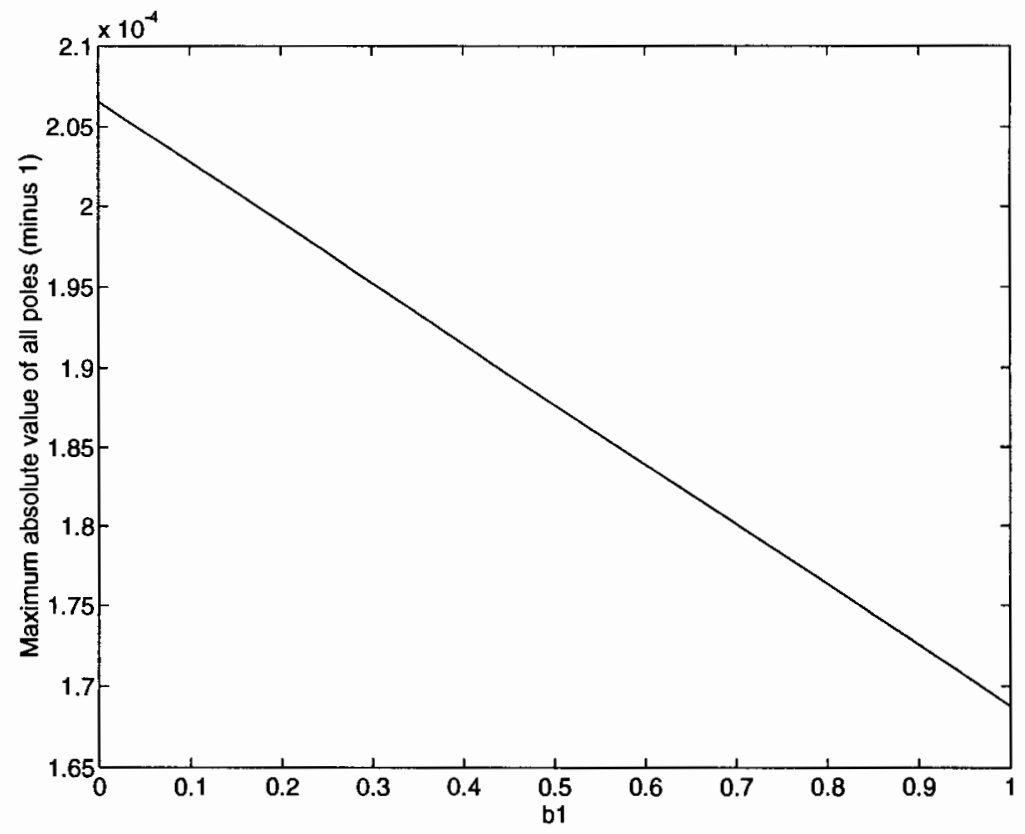

Figure 2: Value of maximum pole minus one. Crank-Nicolson, $\beta=0$. The value is clearly greater than one for all choices of $b_{1}$. 


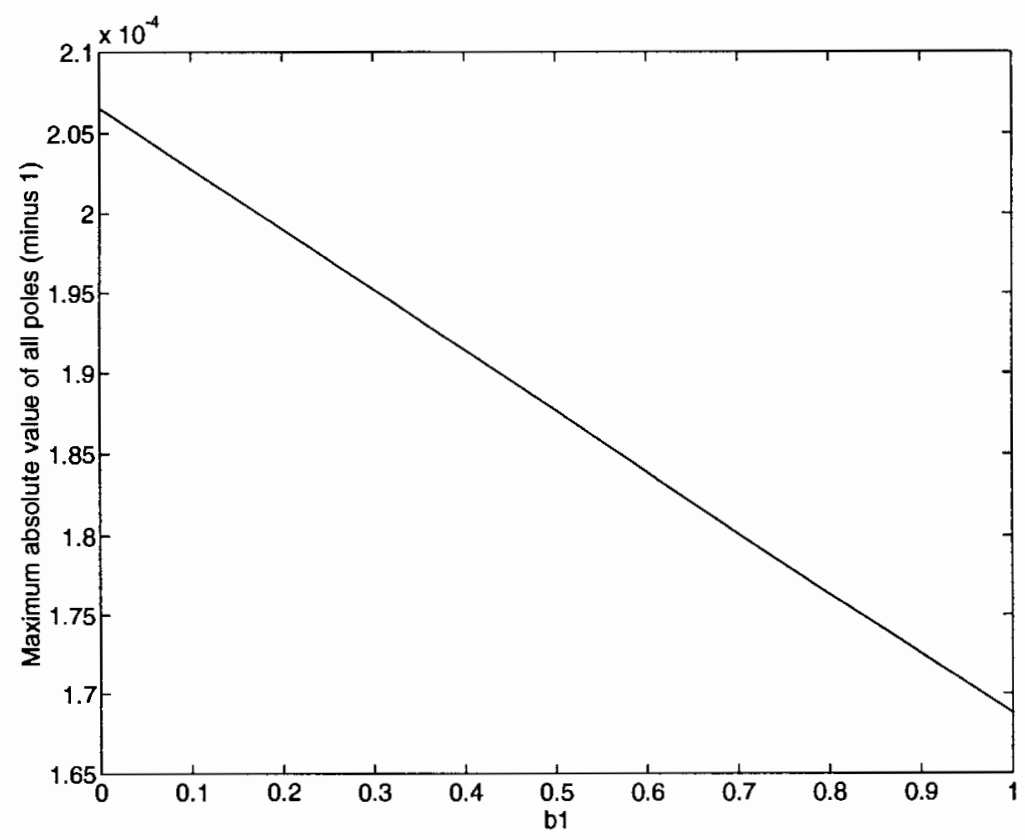

Figure 3: Value of maximum pole minus one. Crank-Nicolson, $\beta=0$. The value is clearly greater than one for all choices of $b_{1}$. 


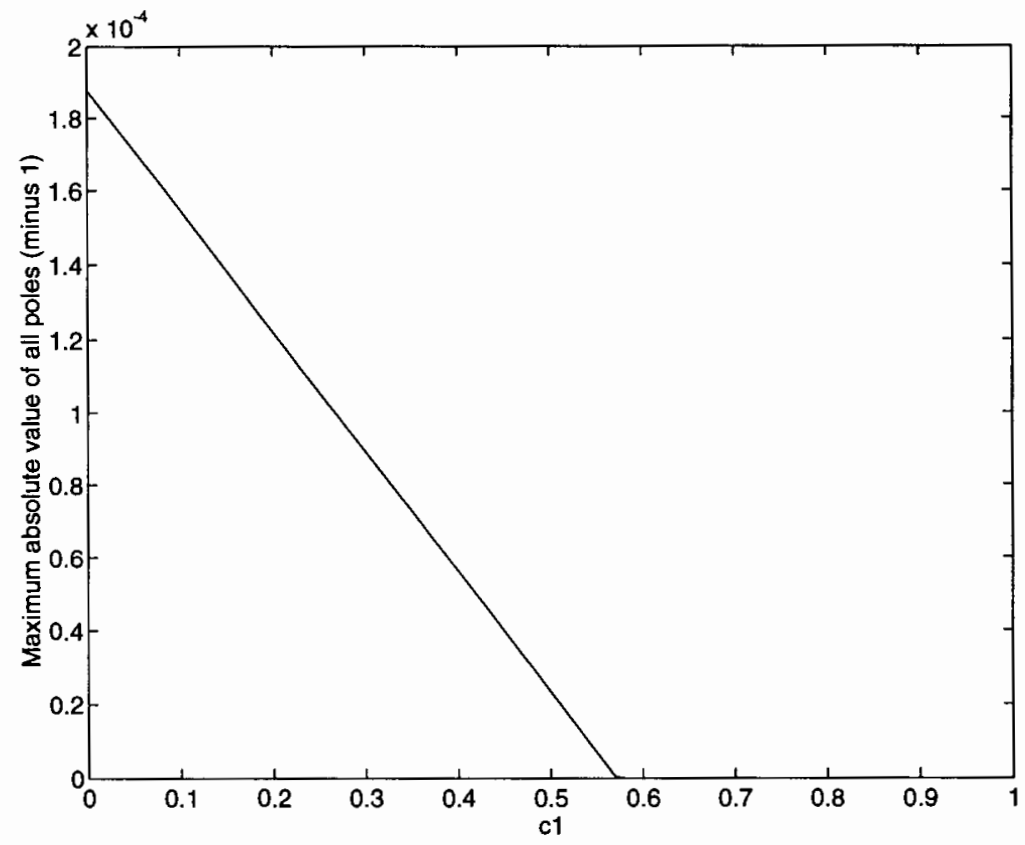

Figure 4: Value of maximum pole minus one. Crank-Nicolson, $\beta=0$. The scheme becomes stable for $c_{1}$ greater than approximately $0.55<c_{1}$. 


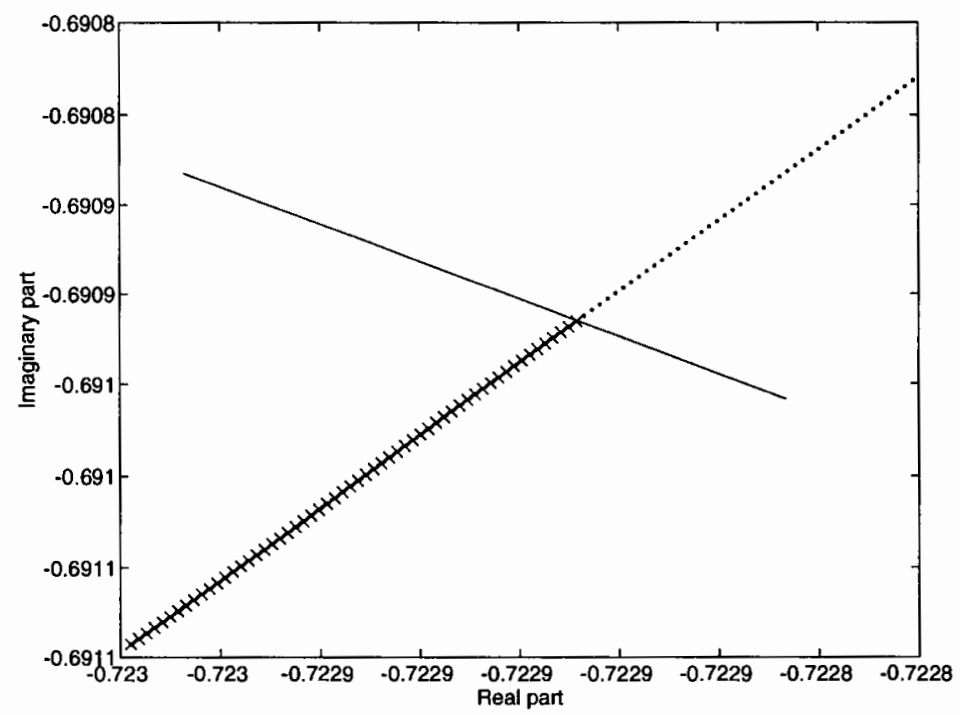

Figure 5: The position of the maximum pole and the pole corresponding to the largest wavenumber. They coincide for small values of $c_{1}$. For large values of $c_{1}$ the largest pole corresponds to wavenumber $k=0$ and is located at $1+0 i$. Dots and crosses conicides until the scheme is stable. Solid - Part of the unitcircle. Crosses - The position of the largest pole (if inside scale of plot). Dots - Position of pole corresponding to the largest wavenumber. 


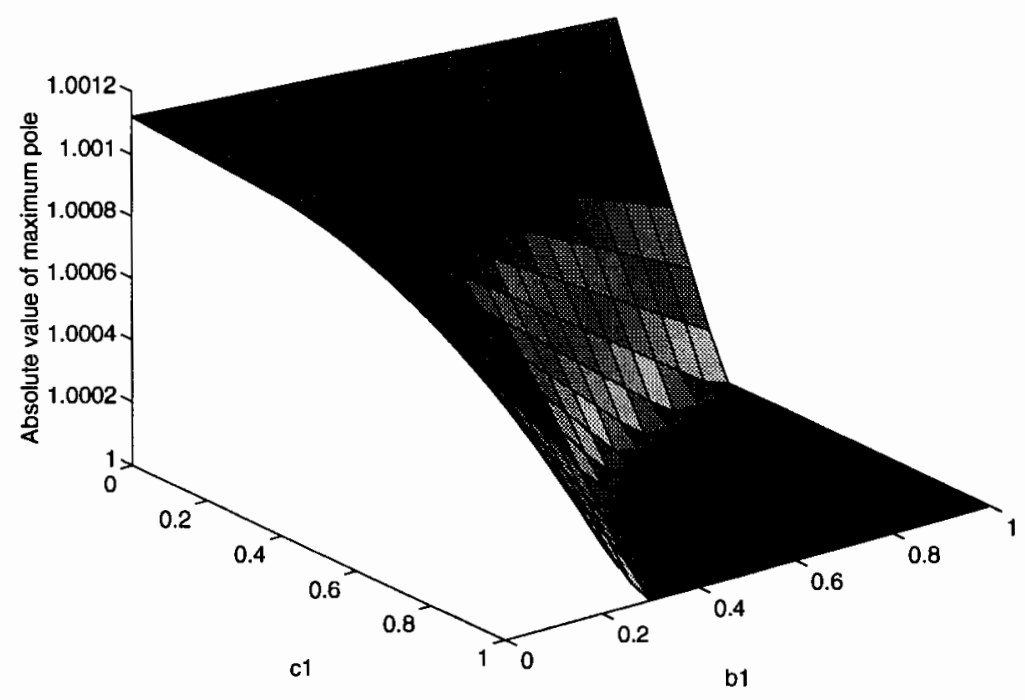

Figure 6: Absolute value of maximum pole for $\beta=0, a=0.5, b_{2}=1, c_{2}=1$ and varying $b_{1}$ and $c_{1}$. Stable scheme occurs for large values of $c_{1}$ and $b_{1}$. 


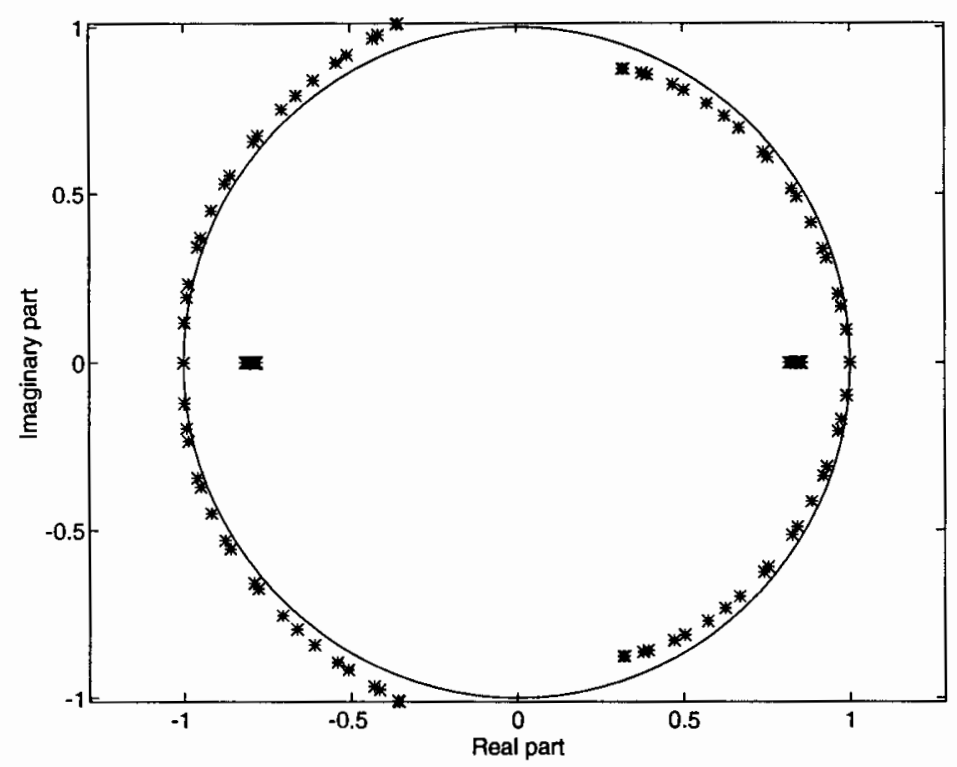

Figure 7: Position of poles for $\beta=1$ schemes, for a large value of the attenuation parameter $\tau$. Clearly unstable. It is always the poles close to -1 , which are outside the unit circle for the unstable schemes.

Two schemes $\left(b_{1}=0, c_{1}=1\right)$ and $\left(b_{1}=1, c_{1}=0\right)$ are possible to implement as staggered schemes. One with the memory variable $r$ located at the same positions as the pressure $p$ or located at the same positions as the velocity $v$. Figure 8 suggests that schemes close to one of the staggered schemes is conditionally stable. The same conclusion could be made from the pole plot for $\beta=0$, Figure 6 , even though it is not as clear as in Figure 8 , since the staggered schemes can only be partially constructed for $\beta=0$.

The numerical dispersion properties of staggered schemes are better than non-staggered schemes', which make a staggered formulation more prefferable than a non-staggered. The most efficient (least necessary amount of computation) staggered formulation places the memory variable $r$ at the location of pressure $p$. This is the most efficient scheme, which is second order accurate in time and fourth order accurate in space, where the memory variable is updated in time by an unconditionally stable scheme (Crank-Nicolson). 


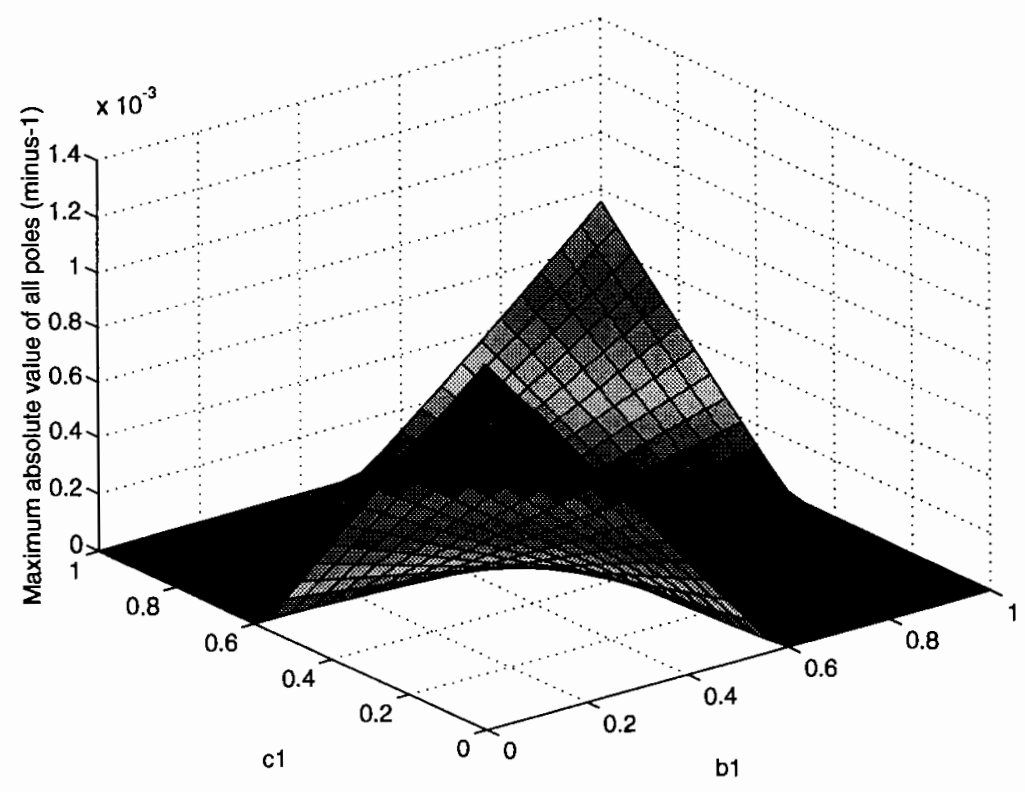

Figure 8: Absolute value of maximum pole for $\beta=1, a=0.5, b_{2}=c_{2}=0.5$ and varying $b_{1}$ and $c_{1}$. Stable schemes occur for (equivalent) staggered stencils and schemes with coefficients approaching the staggered scheme configuration. 


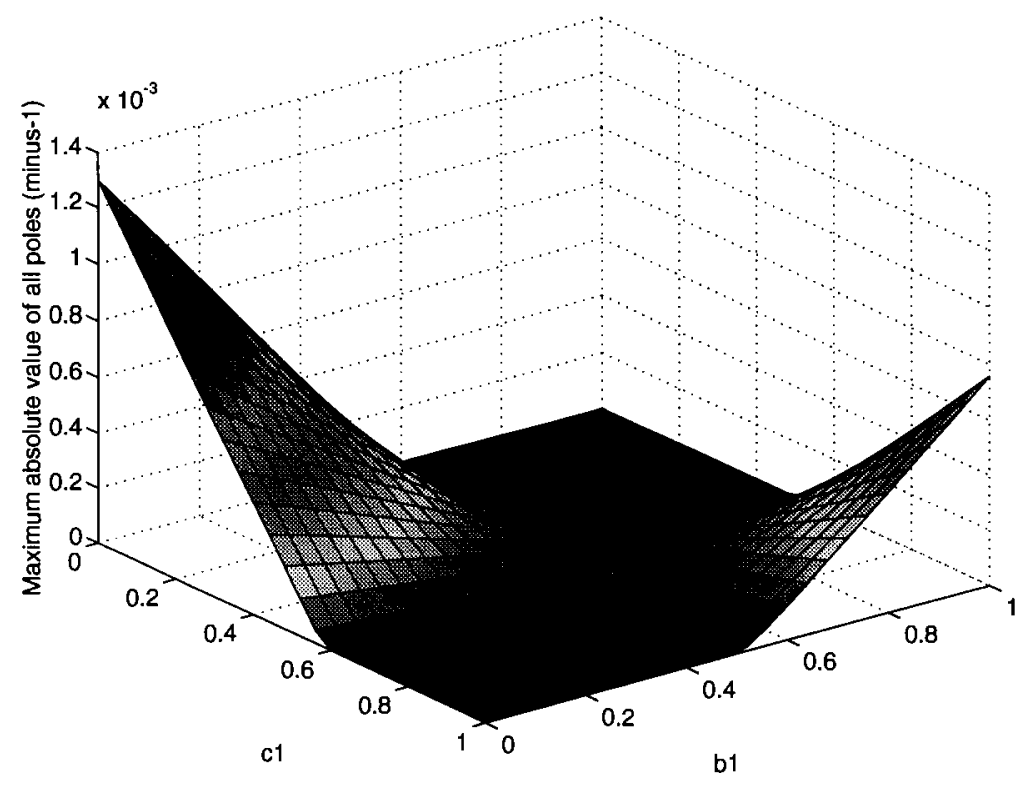

Figure 9: Absolute value of maximum pole for $\beta=1, a=0.5, b_{2}=c_{2}=0.5$ and varying $b_{1}$ and $c_{1}$. Stable schemes occur for (equivalent) staggered stencils and schemes with coefficients approaching the staggered scheme configuration. Other view. 


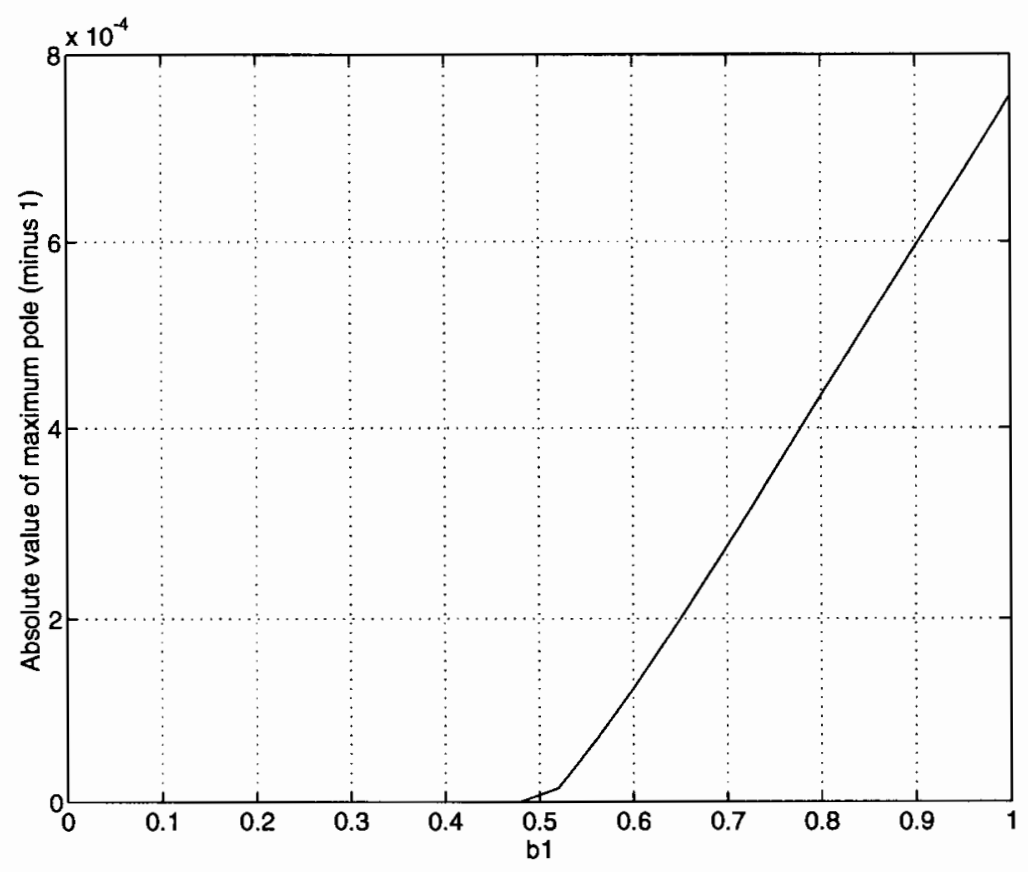

Figure 10: Value of maximum pole minus one. Crank-Nicolson, $\beta=1$. The scheme becomes unstable for $b_{1}$ greater than approximately $0.47<b_{1}$. 


\section{Stability Condition for the Staggered Scheme}

It is necessary to know the stability condition of a finite difference scheme in order to use it to solve a differential equation. Hence, the stability condition of the scheme suggested in the section above must be found. The $Z$ - and Fourier transform of the discrete staggered formulation of the viscoelastic differential equation (1) yields the following third order equation, [3], [4], and [9]. The corresponding sixth order equation, Equation (3), contains only even order terms and is hence equivalent to a third order equation.

$$
\begin{aligned}
& z^{3}+z^{2}\left[-q-2+\psi^{2}\left(1+\tau\left(1-q_{r}\right)\right)\right]+ \\
& z\left[1+2 q-q \psi^{2}\left(1+\tau\left(1+q_{r} / q\right)\right)\right]-q \\
& =0
\end{aligned}
$$

where $q=\frac{2-\eta}{2+\eta}, q_{r}=\frac{\eta}{2+\eta}$, and $\psi=2 \lambda\left(\frac{\sin \left(k_{0} / 2\right)\left(13-\cos \left(k_{0}\right)\right)}{12}\right) . \psi$ is slightly different from before due to the staggered grid. There are a few simple limiting cases where the solution easily can be found. If $\eta \rightarrow 0$ then $z_{1,2}=$ $1-\psi^{2}(1+\tau) / 2 \pm \sqrt{\left[1-\psi^{2}(1+\tau) / 2\right]^{2}-1}, z_{3}=1$, and for $k_{0}=0, z_{1,2}=1$ and $z_{3}=q$. For a corresponding acoustic scheme the roots would be $z_{1,2}=$ $1-\psi^{2} / 2 \pm \sqrt{\left[1-\psi^{2} / 2\right]^{2}-1}$. This is suggests a likely stability limit at either $\psi \leq 2$ or $\psi \sqrt{1+\tau} \leq 2$. The limits correspond to

$$
\lambda \frac{7}{6} \leq 1
$$

where $\lambda$ could be either

$$
\lambda=\frac{c_{0} \Delta t}{\Delta x}
$$

or

$$
\lambda=\frac{c_{\max } \Delta t}{\Delta x}=\frac{c_{0} \sqrt{1+\tau} \Delta t}{\Delta x} .
$$

The number $c \Delta t / \Delta x$ is usually referred to as the Courant number.

The stability limit is most easily investigated numerically by fixing the value for $c_{0}$ and $\Delta x$ and varying $\Delta t$. Figure 11 shows the absolute value of the maximum pole and the absolute value of a complex pole for the largest possible wavenumber (corresponding to spatial Nyquist frequency), where $\tau=0$ and $\eta \rightarrow 0$. The two expressions, Equation (6) and (7), for $\lambda$ yield 


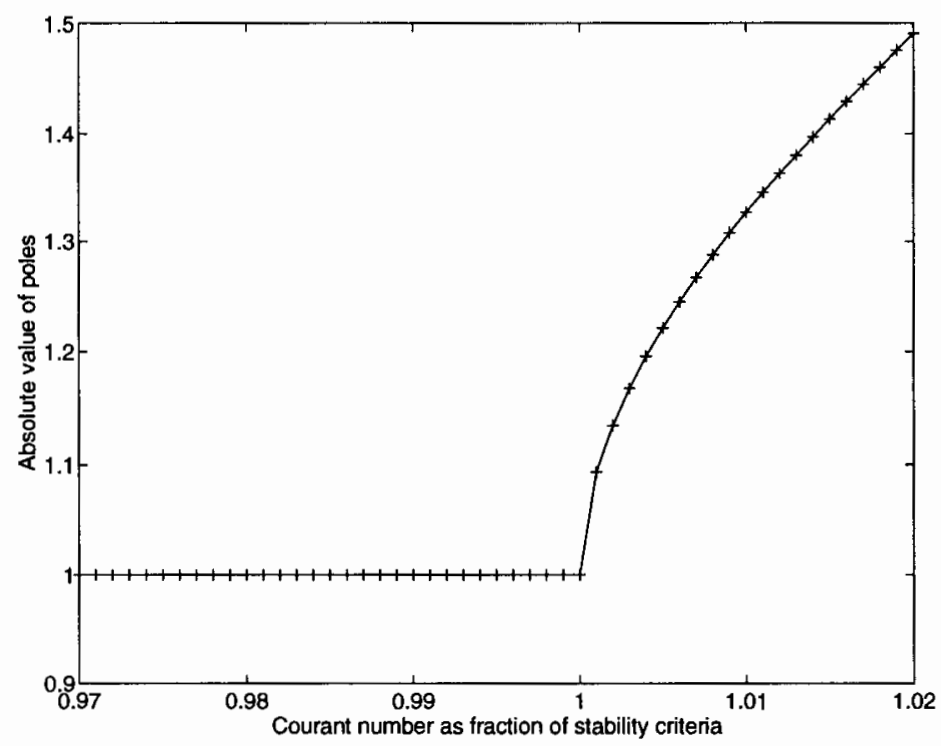

Figure 11: Absolute value of maximum pole and absolute value of pole corresponding to maximum wavenumber. The poles become larger than one exactly at the stability limit. $\tau=0, \eta \rightarrow 0$. Solid - Absolute value of pole correspoding to maximum wavenumber. Plus - The absolute value of the pole with the largest absolute value of all poles for all wavenumbers.

the same stability limit for $\tau=0$, which is a purely acoustic medium. Using expression $(6)$ for the stability limit for $\tau=0.02(Q \approx 100), \eta \rightarrow 0$, and plotting the same poles shows that the acoustic stability limit is larger than the actual, Figure 12. Plotting the same poles but using the expression (7) as the stability limit results in Figure 13. The poles become larger than one exactly at the stability limit in Equation (7).

The numerical stability results supports the theory for $\eta \rightarrow 0$. In real simulations, however, $\eta$ will be a finite value, since otherwise the simulations will essentially be acoustic with very little viscous influences unless $\tau$ is very large $(\tau>1)$. Solving Equation (4) for $\eta=0.2$ and $\tau=0.02$ yields the pole configuration in Figure 14. The stability limit seems to be the same for a finite value of $\eta$. Increasing $\tau$ to $\tau=0.2$ has no effect on the stability limit, yet is the configuration changed, Figure 15. It is possible to see in Figure 15 that the absolute value of the pole corresponding to the maximum wavenumber starts moving towards one (the unitcircle) for lower wavenumbers than in 


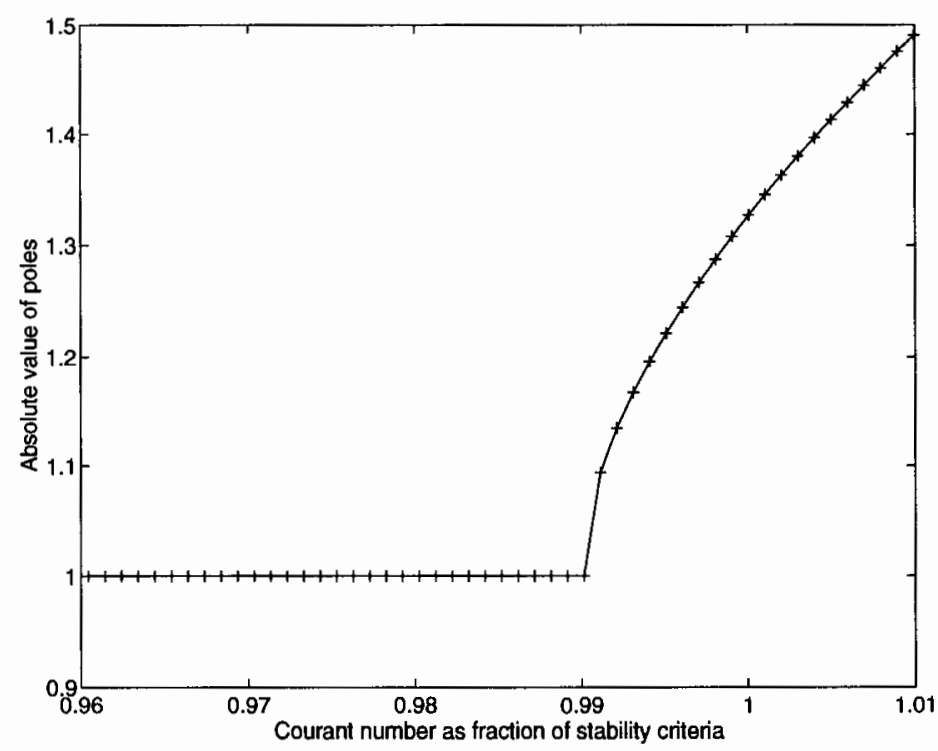

Figure 12: Absolute value of maximum pole and absolute value of pole corresponding to maximum wavenumber. The Courant number is compared to the stability limit in Equation (6). The scheme becomes unstable for a Courant number slightly less than that stability limit. $\tau=0.02, \eta \rightarrow 0$. Solid - Absolute value of pole correspoding to maximum wavenumber. Plus - The absolute value of the pole with the largest absolute value of all poles for all wavenumbers. 


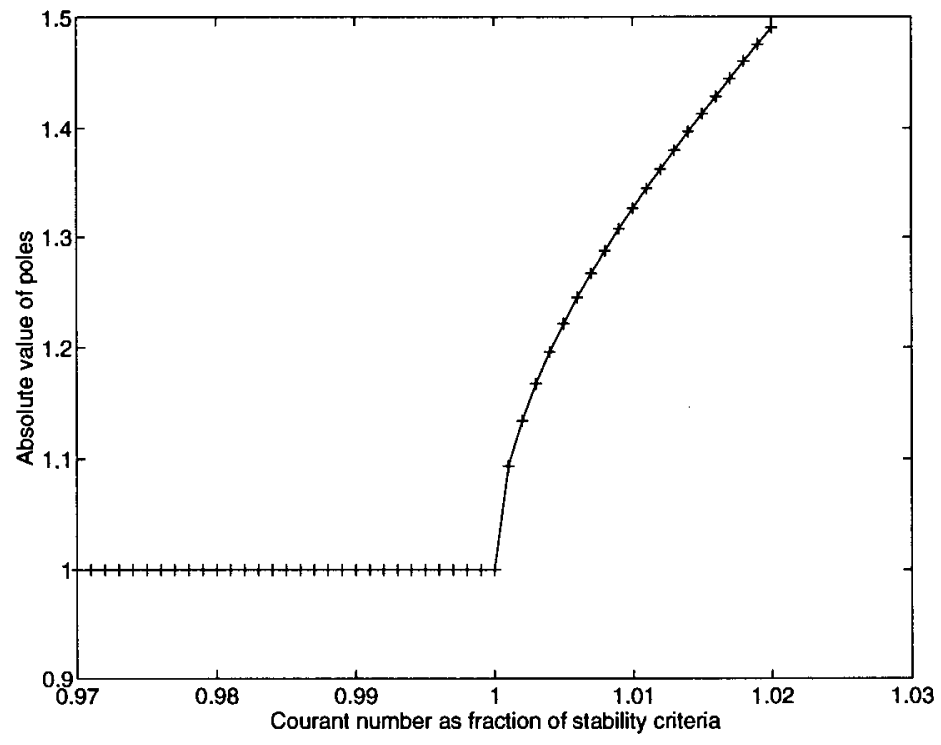

Figure 13: Absolute value of maximum pole and absolute value of pole corresponding to maximum wavenumber. The poles become larger than one exactly at the stability limit in Equation (7). $\tau=0.02, \eta \rightarrow 0$. Solid Absolute value of pole correspoding to maximum wavenumber. Plus - The absolute value of the pole with the largest absolute value of all poles for all wavenumbers. 


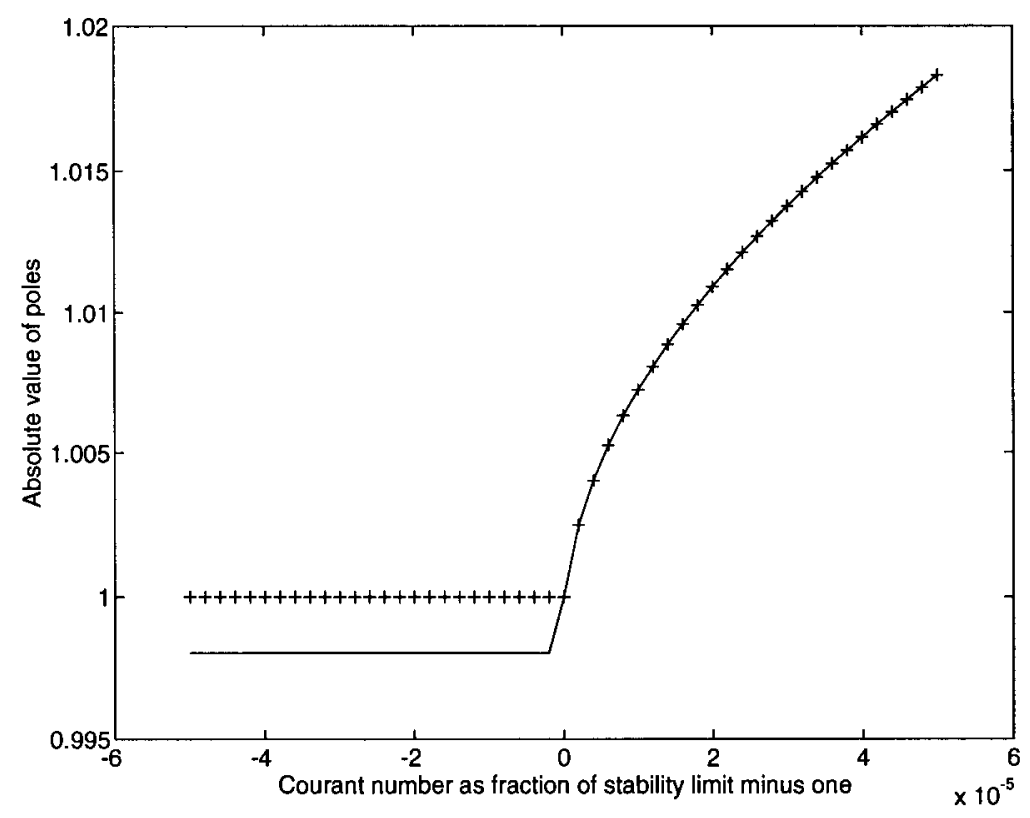

Figure 14: Absolute value of maximum pole and absolute value of pole corresponding to maximum wavenumber. The poles become larger than one exactly at the stability limit in Equation (7). $\tau=0.02, \eta=0.2$. Solid Absolute value of pole correspoding to maximum wavenumber. Plus - The absolute value of the pole with the largest absolute value of all poles for all wavenumbers.

Figure 14. For $\eta=5$ Figure 16 suggests the same stability limit as before. The specific behavior of the poles is naturally different from before.

The exact pole locations for a range of wavenumbers are for $\tau=0.5$, $\eta=0.2$ shown in Figure 17 (stable) and 18 (unstable). The complex poles corresponding to propagating modes become real for $\psi$ large enough to violate the stability condition. One of the poles attains an absolute value larger than one whereas the other has an absolute value less than one. Figures 19 and 20 show corresponding plots for $\eta=5(\tau=0.5)$. Here the originally real pole breaks out of the unit circle in contrast to the $\eta=0.2$ case. 


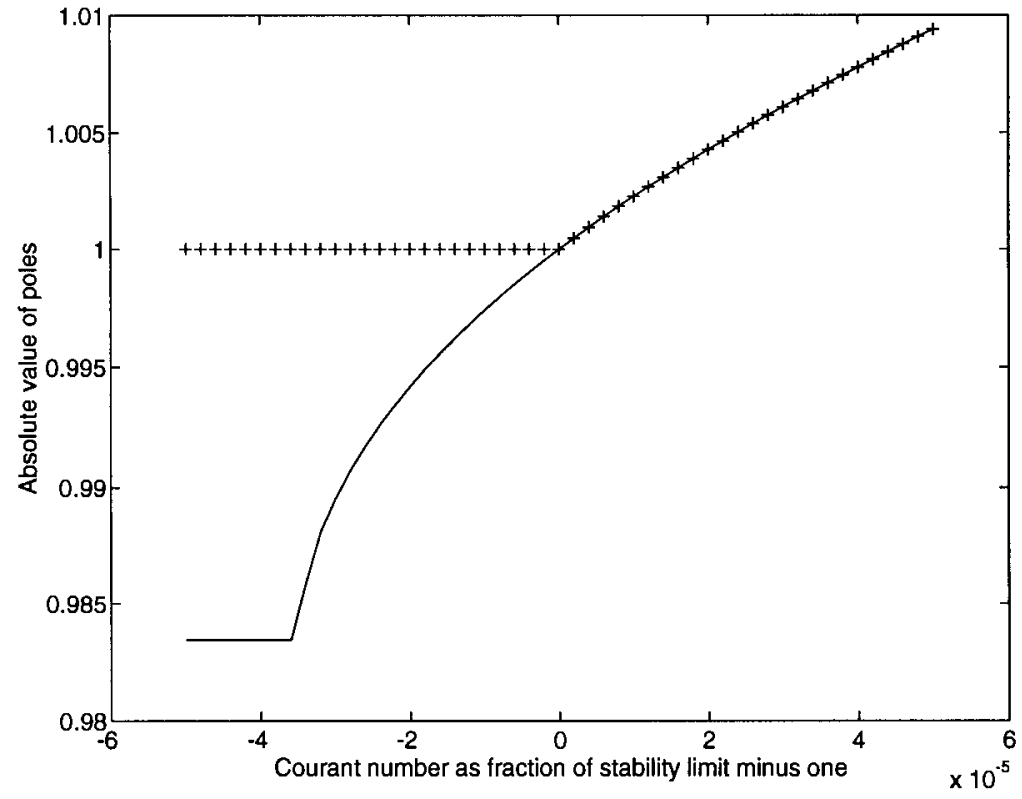

Figure 15: Absolute value of maximum pole and absolute value of pole corresponding to maximum wavenumber. The poles become larger than one exactly at the stability limit in Equation (7). $\tau=0.2, \eta=0.2$. Solid Absolute value of pole correspoding to maximum wavenumber. Plus - The absolute value of the pole with the largest absolute value of all poles for all wavenumbers. 


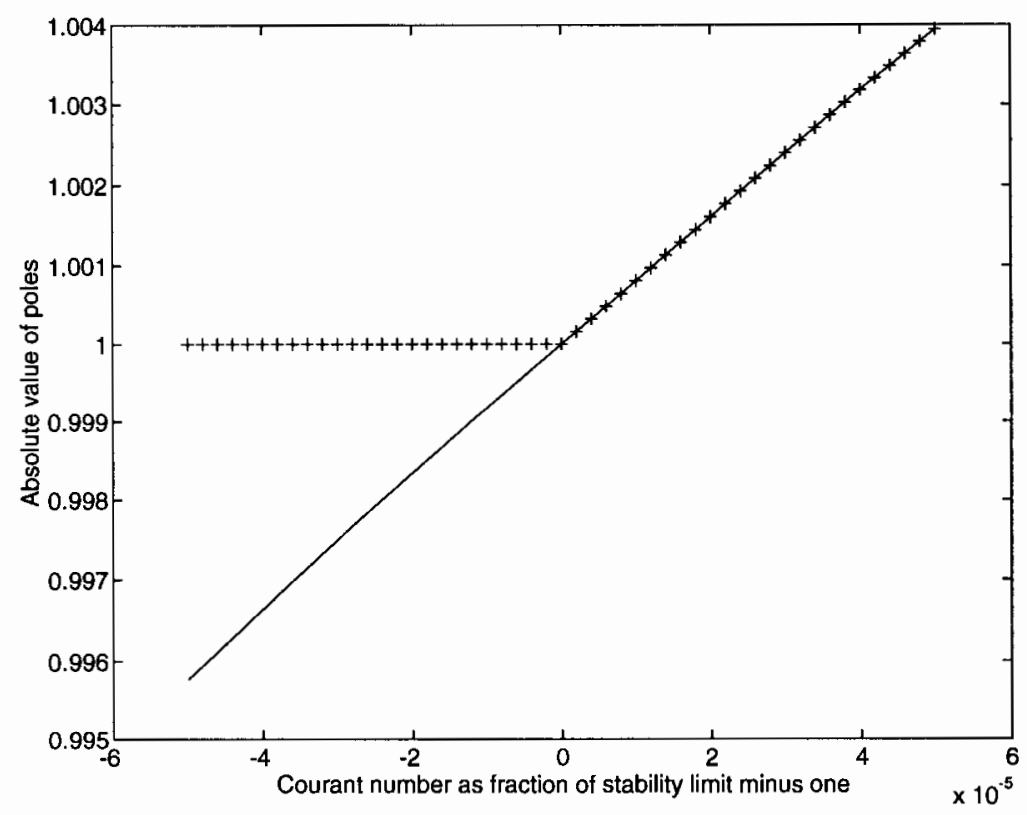

Figure 16: Absolute value of maximum pole and absolute value of pole corresponding to maximum wavenumber. The poles become larger than one exactly at the stability limit in Equation (7). $\tau=0.02, \eta=5$. Solid Absolute value of pole correspoding to maximum wavenumber. Plus - The absolute value of the pole with the largest absolute value of all poles for all wavenumbers. 


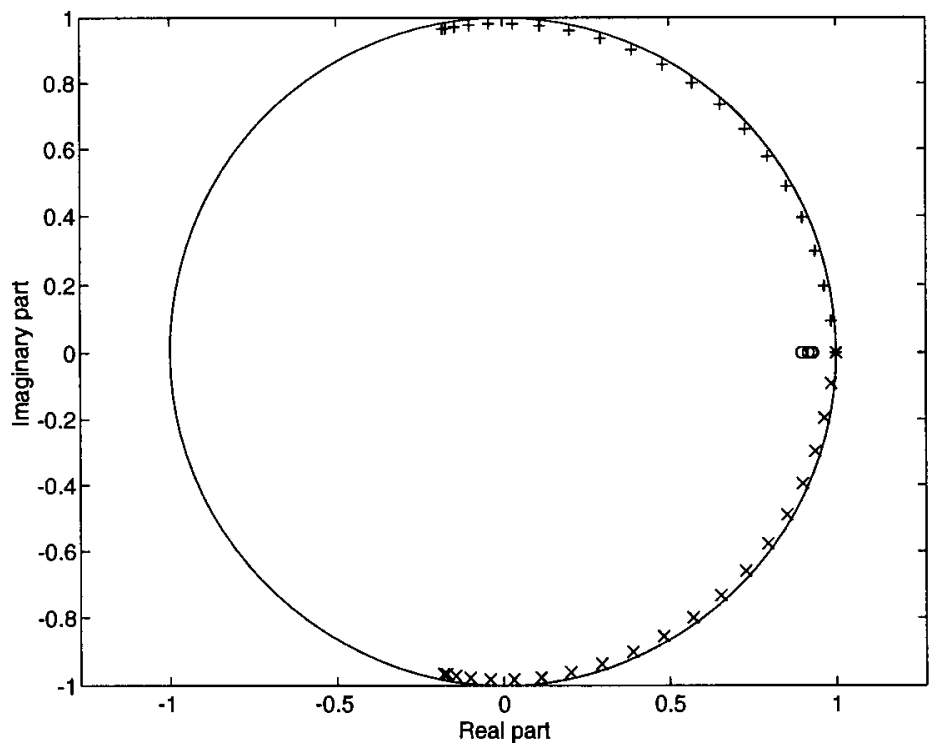

Figure 17: Location of poles (roots) for the Courant number below the stability limit, $\eta=0.2$ and $\tau=0.5$. Two sets of poles closely follow the unit circle, whereas a purely real pole changes value around $q$. 


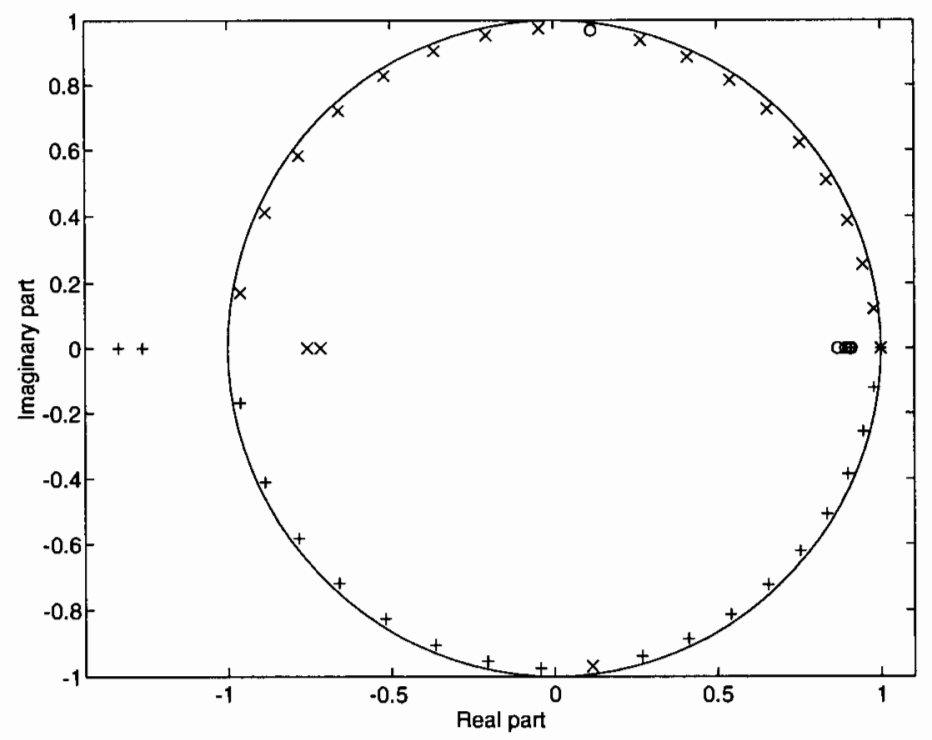

Figure 18: Location of poles (roots) for the Courant number above the stability limit, $\eta=0.2$ and $\tau=0.5$. Two sets of poles closely follow the unit circle, whereas a purely real pole changes value around $q$. The poles following the unit circle pass for some wavenumber the point $-1+0 i$ and both become purely real. One with an absolute value greater than one and one less than one. The modes corresponding to wavenumbers where the poles are greater than one will numerically explode. 


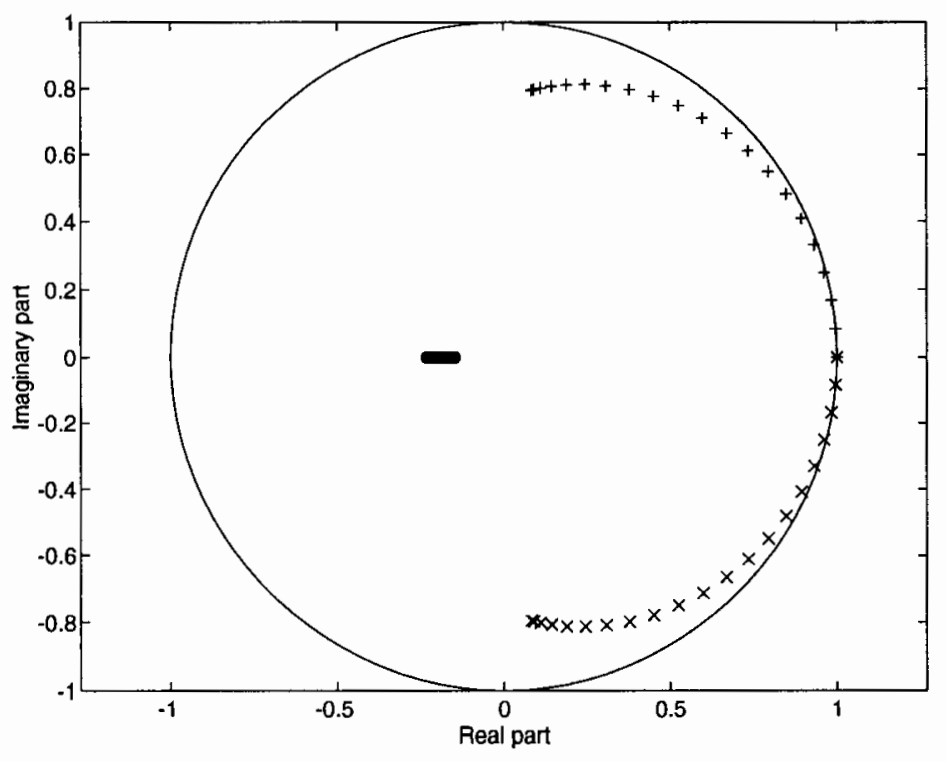

Figure 19: Location of poles (roots) for the Courant number below the stability limit, $\eta=5$ and $\tau=0.5$. Two sets of poles closely follow the unit circle for small wavenumbers but have considerably smaller absolute value for large wavenumbers. A purely real pole changes value around $q$. Here $q$ is negative. 


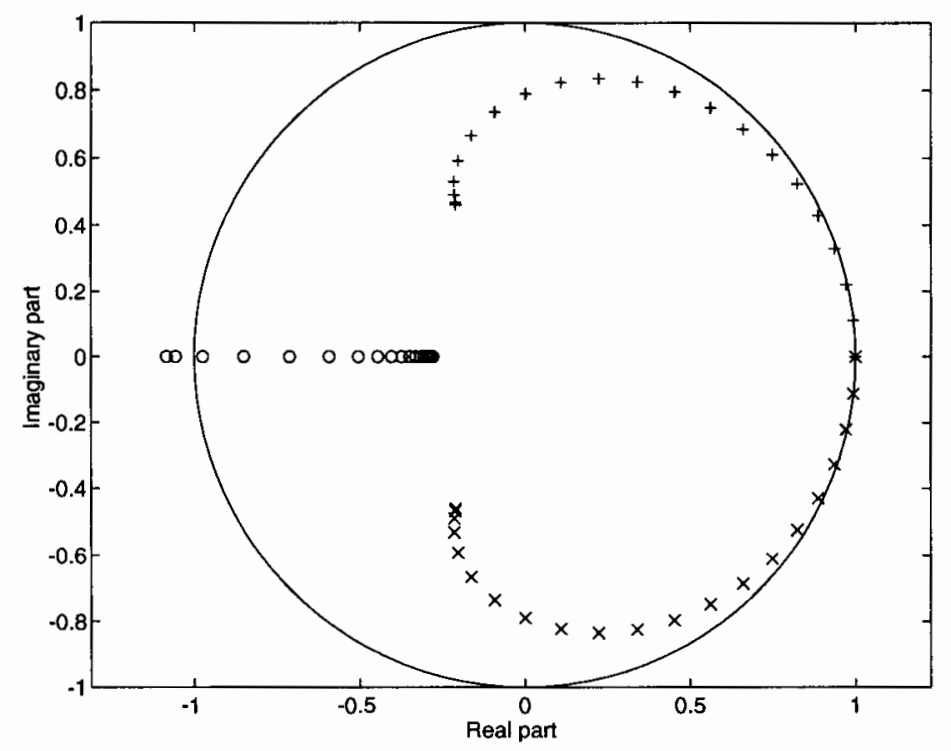

Figure 20: Location of poles (roots) for the Courant number above the stability limit, $\eta=5$ and $\tau=0.5$. Two sets of poles closely follow the unit circle for small wavenumbers but have considerably smaller absolute value for large wavenumbers. A purely real pole dcreases its value from $q$, which is its value for 0 wavenumber. The purely real pole passes for some wavenumber the point $-1+0 i$ and attains an absolute value greater than one. This in contrast to the case $\eta=0.2$ where the complex poles became real and their absolute greater than one. The modes corresponding to wavenumbers where the pole is greater than one will numerically explode. 


\section{Conclusions}

Stability of finite difference schemes is difficult to predict. The poles of the schemes must be analyzed for all possible parameter combinations. Several schemes can be investigated simultaneously by deriving the modal equation for a set of general parameters.

Several conditionally stable finite difference scheme were found for the viscoelastic wave equation corresponding to a constitutive relation of one standard linear solid. The most numerically efficient scheme is a staggered scheme second order accurate in time and fourth order accurate in space. Only spatially fourth order accurate schemes were investigated.

The stability limit for the staggered scheme is

$$
\frac{c_{\max } \Delta t}{\Delta x} \leq \frac{6}{7 \sqrt{D}},
$$

where $D$ is the dimension and $c_{\max }$ is the infinite frequency velocity. Different poles break out of the unit circle for too large Courant numbers depending on the value $\eta$.

\section{Acknowledgments}

This research was supported by ONR grant N00014-89-J1115, the Schlumberger Foundation, and The Rice Inversion Project. TRIP Sponsors for 1994 are Advance Geophysical, Amoco Production Co., Conoco Inc., Cray Research Inc., Exxon Production Research Co., Interactive Network Technologies, Mobil Research and Development Corp., and Texaco Inc.

\section{References}

[1] J. O. Blanch, J. O. A. Robertsson, and W. W. Symes. Viscoelastic finite difference modeling. Technical Report TR 93-04, Department of Computational and Applied Mathematics, Rice University, 1993.

[2] J. O. Blanch, J. O. A. Robertsson, and W. W. Symes. Viscoelastic finite difference modeling. In R. Kleinman, T. Angell, D. Colton, F. Santosa, 
and I. Stakgold, editors, Mathematical and numerical aspects of wave propagation, pages 69-81. SIAM, 1993.

[3] J. O. Blanch, J. O. A. Robertsson, and W. W. Symes. Viscoelastic finite difference modeling. In Expanded abstracts from the 63rd Ann. Internat. Mtg. of Soc. Expl. Geophys., pages 990-993, 1993.

[4] J. O. Blanch, J. O. A. Robertsson, and W. W. Symes. Viscoelastic finite difference modeling II. Technical Report TR 93-16, Department of Computational and Applied Mathematics, Rice University, 1993.

[5] J. O. Blanch, J. O. A. Robertsson, and W. W. Symes. Modeling of a constant Q: Methodology and algorithm for an efficient and optimally inexpensive viscoelastic technique. Geophysics, 1994. In press.

[6] S. M. Day and J. B. Minster. Numerical simulation of attenuated wavefields using a Padé approximant method. Geophys. J. Roy. Astr. Soc., 78:105-118, 1984.

[7] B. Gustafsson, A. Sjöberg, and L. Abrahamsson. Numerisk Lösning av Differential Ekvationer. Uppsala University, Department of Scientific Computing, 1988.

[8] A. R. Levander. Fourth-order finite-difference P-SV seismograms. Geophysics, 53:1425-1436, 1988.

[9] J. O. A. Robertsson, J. O. Blanch, and W. W. Symes. Viscoelastic finitedifference modeling. Geophysics, 59:1444-1456, 1994. 